\title{
ABSTRACTS.
}

\section{Nature of the Reaction Due to Fluids in Motion.}

A mathematical investigation is given of the laws governing the forces acting upon the walls of conduits and pipe-lines carrying fluids at rest or in motion. As a working approximation the hypothesis is made that over any section of the stream drawn transversely to the mean stream direction at that locality, the distributions of pressure and velocity are uniform.

In the first instance the hydrostatic case is discussed of fluid maintained at rest under a definite pressure head in a ring-shaped vessel of irregular form, constituting a "pipe-line," admitting of bends and constrictions. The pipe is supposed to be situated in vacuo, and to be built with its walls rigid and weightless. Considering the portion of fluid comprised between any two transverse sections of the pipe, the resultant force is computed which must be resisted by the bounding walls.

Passing to the extension of the problem where steady flow may be supposed, maintained by means of a small turbine introduced in the circuit, the resultant of the pressure forces, due to all causes, acting upon the actual walls bounding any length of the circuits, is referred to as the wall-pressure. In this connection it is recalled that in common usage the term "Reaktion" refers to the force system acting upon the walls, which is contributed by dynamic causes alone, no account being taken of the pressure acting over the transverse sections cutting off the pipelength considered. It is estimated as the difference between the wall forces acting when the fluid is in motion and when it is at rest. This standpoint is thought liable to lead to error in certain practical calculations.

Proceeding to the calculation of the wall-pressure, expressions are first derived determining the resultant effect contributed by centrifugal forces due to the curvature of the stream flowing through a given length of pipe. It is inferred that, as regards the form of the expressions derived, the geometrical shape of the length of the pipe is immaterial, and, further, that in the intermediate portion, regions of cavitation are permissible within the fluid, which may be supposed largely filled in with turbulent fluid. Frictional and energy losses which may arise from eddy motion are said to affect the value of the final expressions only in reducing the velocity over the discharge section.

An investigation follows of the terms to be included in the wall-pressure due to causes other than those of centrifugal origin. A special length of the pipe is considered, without curvature, in the form of a truncated cone. Allowing for the acceleration forces which are necessary to produce the requisite velocity changes, the total wall-pressure is computed, both when the axis of the cone is vertical and when it is horizontal. Referring to practical application in this connection, the formulæ obtained are recommended for problems such as the calculation of the strength of screws utilised for attaching outlet chambers of various types of the walls of the conduit. Special reference is made to the cases of a tapering outlet attached at an angle to a plane wall, and of a nozzle whose discharge mouth is cut skew. It is stated that conditions discussed are approximately reproduced within the paddles of turbines.

The forces operating on the walls consequent on curvature are more closely examined. The total wall-reaction, inclusive of centrifugal effects, is investigated for length of curved pipe circuit. It is considered sufficient to examine two special cases, consisting of curved pipes of uniform bore, fitted with conical intake and discharge mouth-pieces respectively.

In the concluding sections reasons are advanced to emphasise the desirability of an improvement and a standardisation of nomenclature in the subject.- $(\mathrm{A}$. Budan "Oesterreichische Flug-Zeitschrift," Nos. I-4, Jan.-Feb., I9I8.) 